\title{
Aluthge transform of operators on the Bergman space
}

Received: 4 June 2019 / Accepted: 29 October 2019 / Published online: 26 November 2019

(C) The Author(s) 2019

\begin{abstract}
The aim of this paper is to explore some sufficient conditions for Aluthge transform of Toeplitz operators on the Bergman space to be unitary, average of unitaries and normal.
\end{abstract}

\section{Mathematics Subject Classification 47B38 - 47B35}

\section{Introduction}

Let $H, K$ be two separable, infinite dimensional, complex Hilbert spaces and let $\mathcal{L}(H)$ denote the algebra of all bounded linear operators on $H$. An arbitrary operator $T$ in $\mathcal{L}(H)$ has a polar decomposition $T=U|T|$, where $|T|=\left(T^{*} T\right)^{\frac{1}{2}}$ and $\mathrm{U}$ is the appropriate partial isometry $\left(\right.$ with $\operatorname{ker}(U)=\operatorname{ker}(T)$ and $\operatorname{ker}\left(U^{*}\right)=\operatorname{ker}\left(T^{*}\right)$ ). The Aluthge transformation $\Delta(T)=|T|^{\frac{1}{2}} U|T|^{\frac{1}{2}}$ was first introduced by Aluthge [2]. An operator $T$ is said to be normal if $T^{*} T=T T^{*}$. The operator $T$ is called quasinormal if $\left(T T^{*}\right) T=\left(T^{*} T\right) T$. An operator $T$ is called hyponormal if $T^{*} T \geq T T^{*}$. An operator $T$ is paranormal if $\|T x\|^{2} \leq\left\|T^{2} x\right\|\|x\|$. Here, we denote $\Delta^{*}(T)=(\Delta(T))^{*}$ for $T \in \mathcal{L}(H)$. Let $\operatorname{sp}(T)$ denotes the spectrum of $T$. Define $m(T)=\inf \{\|T x\|:\|x\|=1\}$. An operator $T$ is called w-hyponormal if $|\Delta(T)| \geq|T| \geq\left|\Delta^{*}(T)\right|$. An operator $T$ is said to be binormal if $\left[|T|,\left|T^{*}\right|\right]=0$, where $[T, S]=T S-S T$. The tensor product of $x \in H$ and $y \in K$ is a conjugate bilinear functional $x \otimes y: H \times K \longrightarrow \mathbb{C}$ defined by $(x \otimes y)(u, v)=\langle x, u\rangle\langle y, v\rangle$ for every $(u, v) \in H \times K$. The collection of all (finite) sums of tensors $x_{i} \otimes y_{i}$ with $x_{i} \in H$ and $y_{i} \in K$, denoted by $H \otimes K$, is a complex linear space equipped with an inner product $\langle.,\rangle:.(H \otimes K) \times(H \otimes K) \longrightarrow \mathbb{C}$ defined for arbitrary $\sum_{i=1}^{N} x_{i} \otimes y_{i}$, and $\sum_{j=1}^{M} w_{j} \otimes z_{j}$ in $H \otimes K$, by $\left\langle\sum_{i=1}^{N} x_{i} \otimes y_{i}, \sum_{j=1}^{M} w_{j} \otimes z_{j}\right\rangle=\sum_{i=1}^{N} \sum_{j=1}^{M}\left\langle x_{i}, w_{j}\right\rangle\left\langle y_{i}, z_{j}\right\rangle$ (the same notation for the inner products on $\mathrm{H}, \mathrm{K}$ and $H \otimes K)$. The tensor product on $H \otimes K$ of two operators $\mathrm{T}$ in $\mathcal{L}(H)$ and $\mathrm{S}$ in $\mathcal{L}(K)$ is the operator $T \otimes S: H \otimes K \longrightarrow H \otimes K$ defined by $(T \otimes S) \sum_{i=1}^{N} x_{i} \otimes y_{i}=\sum_{i=1}^{N} T x_{i} \otimes S y_{i}$ for every $\sum_{i=1}^{N} x_{i} \otimes y_{i} \in H \otimes K$, which lies in $\mathcal{L}(H \otimes K)$. The complete inner product space $H \otimes K$ is denoted by $H \hat{\otimes} K$, which is the tensor product space of $\mathrm{H}$ and $\mathrm{K}$. The extension of $T \otimes S$ over the Hilbert space

C. Padhy $\cdot$ S. K. Paikray

Department of Mathematics, Veer Surendra Sai University of Technology, Burla, Odisha 768018, India

E-mail: chinmayee.padhy83@gmail.com

S. K. Paikray

E-mail: skpaikray_math@vssut.ac.in

P. K. Jena $(\varangle)$

P. G. Department of Mathematics, Berhampur University, Bhanjabihar, Berhampur, Ganjam, Odisha 760007, India

E-mail: pabitramath@gmail.com 
$H \hat{\otimes} K$ denoted by $T \hat{\otimes} S$ is the tensor product of $\mathrm{T}$ and $\mathrm{S}$ on the tensor product space, which lies in $\mathcal{L}(H \hat{\otimes} K)$ (see [6], [12] and [17]).

Definition 1.1 If the sequence

$$
\left\{\cdots, T^{3}\left(T^{3}\right)^{*}, T^{2}\left(T^{2}\right)^{*}, T T^{*}, T^{*} T,\left(T^{2}\right)^{*} T^{2},\left(T^{3}\right)^{*} T^{3},\left(T^{4}\right)^{*} T^{4}, \cdots\right\}
$$

is commutative, the operator $T \in \mathcal{L}(H)$ is called centered operator (see [10]).

Definition 1.2 A bounded linear operator $T$ is said to be Fredholm operator if and only if its range is closed, $\operatorname{dim} \operatorname{ker} T$ and $\operatorname{dim} \operatorname{ker} T^{*}$ are finite.

The index of an operator $T \in \mathcal{L}(H)$ is a function from $\mathcal{F}(H)$ to $\mathbb{Z}$ which is denoted by $i(T)$ and defined by $i(T)=\operatorname{dim} \operatorname{ker} T-\operatorname{dim} \operatorname{ker} T^{*}$, where $\mathcal{F}(H)$ is a collection of fredholm operators on $H$ (see [14]). An operator $T$ is normaloid if $\|T\|=\sup \{|\langle T x, x\rangle| ;\|x\|=1\}$.

The Bergman space $A^{2}(\mathbb{D})$ is the Hilbert space consisting of all analytic functions $f$ on the open unit disk $\mathbb{D}=\{z \in \mathbb{C}:|z|<1\}$ for which

$$
\|f\|_{A^{2}(\mathbb{D})}=\left(\int_{\mathbb{D}}|f(z)|^{2} d A(z)\right)^{\frac{1}{2}}<\infty,
$$

where $d A(z)$ is the Lebesgue area measure on the open unit disk $\mathbb{D}$, normalized so that the measure of the disk $\mathbb{D}$ equals 1 . If $h(z)=\sum_{n=0}^{\infty} a_{n} z^{n}$ and $k(z)=\sum_{n=0}^{\infty} b_{n} z^{n}$ are two functions in $A^{2}(\mathbb{D})$, then the inner product of $h$ and $k$ is given by

$$
\langle h, k\rangle=\int_{\mathbb{D}} h(z) \overline{k(z)} d A(z)=\sum_{n=0}^{\infty} \frac{a_{n} \overline{b_{n}}}{n+1} .
$$

For $z \in \mathbb{D}$, the Bergman reproducing kernel is the function $K_{z} \in A^{2}(\mathbb{D})$ such that $f(z)=\left\langle f, K_{z}\right\rangle$ for every $f \in A^{2}(\mathbb{D})$. The normalized reproducing kernel $k_{z}$ is the function $\frac{K_{z}}{\left\|K_{z}\right\|_{2}}$. For any $n \geq 0, n \in \mathbb{Z}$, let $e_{n}(z)=\sqrt{n+1} z^{n}$. Then $\left\{e_{n}\right\}$ forms an orthonormal basis for $A^{2}(\mathbb{D})$. For $\phi \in L^{\infty}(\mathbb{D})$, the Toeplitz operator $T_{\phi}$ with symbol $\phi$ is the operator on $A^{2}(\mathbb{D})$ defined by $T_{\phi} f=P(\phi f)$; here, $P$ is the orthogonal projection from $L^{2}(\mathbb{D}, d A)$ onto $A^{2}(\mathbb{D})$.

Let $\overline{A^{2}(\mathbb{D})}$ be the space of conjugate analytic functions in $L^{2}(\mathbb{D}, d A)$. Then, $\overline{A^{2}(\mathbb{D})}=\left\{\bar{g}: g \in A^{2}(\mathbb{D})\right\}$ is closed in $L^{2}(\mathbb{D}, d A)$. Let $\phi \in L^{\infty}(\mathbb{D})$, the little Hankel operator $h_{\phi}: A^{2}(\mathbb{D}) \rightarrow \overline{A^{2}(\mathbb{D})}$ be defined by $h_{\phi} f=\bar{P}(\phi f), f \in A^{2}(\mathbb{D})$ where $\bar{P}$ is the orthogonal projection from $L^{2}(\mathbb{D}, d A)$ onto $\overline{A^{2}(\mathbb{D})}$. There are also numerous equivalent ways of defining little Hankel operators on $A^{2}(\mathbb{D})$. For illustration, define the map $S_{\phi}: A^{2}(\mathbb{D}) \rightarrow A^{2}(\mathbb{D})$ by $S_{\phi} f=P J(\phi f)$, where $J$ is self-adjoint, unitary mapping from $L^{2}(\mathbb{D}, d A)$ into itself given by $J h(z)=h(\bar{z})$. Observe that $J S_{\phi}=h_{\phi}$. So $S_{\phi}$ is unitarily equivalent to $h_{\phi}$.

Let $A u t(\mathbb{D})$ be the Lie group of all automorphisms (biholomorphic mappings) of $\mathbb{D}$. We can define for each $a \in \mathbb{D}$, an automorphism $\phi_{a}$ in Aut $(\mathbb{D})$ such that

(i) $\left(\phi_{a} \circ \phi_{a}\right)(z) \equiv z$;

(ii) $\phi_{a}(0)=a, \phi_{a}(a)=0$;

(iii) $\phi_{a}$ has a unique fixed point in $\mathbb{D}$.

In fact, $\phi_{a}(z)=\frac{a-z}{1-\bar{a} z}$ for all $a$ and $z$ in $\mathbb{D}$. It is easy to verify that the derivative of $\phi_{a}$ at $z$ is equal to $-k_{a}(z)$. It implies the real Jacobian determinant of $\phi_{a}$ at $z$ is $J_{\phi_{a}(z)}=\left|k_{a}(z)\right|^{2}=\frac{\left(1-|a|^{2}\right)^{2}}{|1-\bar{a} z|^{4}}$. Given $a \in \mathbb{D}$ and $f$ (any measurable function on $\mathbb{D}$ ), let us define a function $U_{a} f$ on $\mathbb{D}$ by $U_{a} f(w)=k_{a}(w) f\left(\phi_{a}(w)\right)$. Notice that $U_{a}$ is a bounded linear operator on $L^{2}(\mathbb{D}, d A)$ and $A^{2}(\mathbb{D})$ for all $a \in \mathbb{D}$. Further, it can be verified that $U_{a}^{2}=I$, the identity operator, $U_{a}^{*}=U_{a}, U_{a}\left(A^{2}(\mathbb{D})\right) \subset A^{2}(\mathbb{D})$ and $U_{a}\left(\left(A^{2}(\mathbb{D})\right)^{\perp}\right) \subset\left(A^{2}(\mathbb{D})\right)^{\perp}$ for all $a \in \mathbb{D}$. Thus, $U_{a} P=P U_{a}$ for all $a \in \mathbb{D}$ (see [20]). Let $H^{\infty}(\mathbb{D})$ denote the space of bounded analytic functions on $\mathbb{D}$.

In this article, we establish some sufficient conditions for Aluthge transform of Toeplitz operators and elementary operators on Bergman space to be unitary and average of unitaries. Furthermore, we explore some sufficient conditions for Aluthge transform of tensor product of Toeplitz operators to be average of unitaries on the tensor product of Bergman spaces. Again, we explain some sufficient conditions on $T_{\phi}$ and $S_{\psi}$ which imply $\Delta\left(T_{\phi} \hat{\otimes} S_{\psi}\right)$ is normal. 


\section{Unitary operator}

This section contains a few sufficient conditions by which Aluthge transform of Toeplitz operators on $A^{2}(\mathbb{D})$ express as an average of unitaries.

Theorem 2.1 Let $\phi \geq 0$ with $\|\phi\|_{\infty} \leq 1$ for $\phi \in L^{\infty}(\mathbb{D})$. If $\left\|T_{1+\phi}\right\|<1$. Then, the Aluthge transform $\Delta\left(T_{\phi}\right)$ of $T_{\phi}$ on $A^{2}(\mathbb{D})$ can be represented as an average of two unitary operators.

Proof Since $\phi$ is positive, $T_{\phi} \geq 0$ on $A^{2}(\mathbb{D})$. Then, by [1], we obtain $\left\|V-T_{\phi}\right\| \leq\left\|I+T_{\phi}\right\|=\left\|T_{1+\phi}\right\|<1$, where $V$ is any unitary operator on $A^{2}(\mathbb{D})$. Since $\left\|V-T_{\phi}\right\|<1$, so it implies $\left\|I-V^{*} T_{\phi}\right\|<1$. Hence, $V^{*} T_{\phi}$ and $T_{\phi}$ are invertible. Then, by ([13], Lemma 1.1), $\Delta\left(T_{\phi}\right)$ is invertible. Let $\Delta\left(T_{\phi}\right)=U\left|\Delta\left(T_{\phi}\right)\right|$ be the polar decomposition of $\Delta\left(T_{\phi}\right)$. As $\Delta\left(T_{\phi}\right)$ is invertible, the partial isometry $U$ becomes a unitary operator. But from ([13], Proposition 1.6), we obtain $\left\|\Delta\left(T_{\phi}\right)\right\| \leq\left\|T_{\phi}\right\| \leq 1$. This implies $\left\|\left|\Delta\left(T_{\phi}\right)\right|\right\| \leq 1$. Therefore, $I-\left|\Delta\left(T_{\phi}\right)\right|^{2} \geq 0$ and $\left\|I-\left|\Delta\left(T_{\phi}\right)\right|^{2}\right\| \leq 1$. Now, we define two operators as $W_{1}=\left|\Delta\left(T_{\phi}\right)\right|+i\left(I-\left|\Delta\left(T_{\phi}\right)\right|^{2}\right)^{\frac{1}{2}}$ and $W_{2}=\left|\Delta\left(T_{\phi}\right)\right|-i\left(I-\left|\Delta\left(T_{\phi}\right)\right|^{2}\right)^{\frac{1}{2}}$ such that $W_{1}^{*}=W_{2}$. Therefore, it is clear that $W_{1} W_{1}^{*}=I$ and $W_{1}^{*} W_{1}=I$. Thus, $W_{1} W_{1}^{*}=W_{1}^{*} W_{1}=I$ and $W_{2} W_{2}^{*}=W_{2}^{*} W_{2}=I$. This implies that $W_{1}$ and $W_{2}$ are two unitary operators on $A^{2}(\mathbb{D})$. Hence, $\Delta\left(T_{\phi}\right)=U\left|\Delta\left(T_{\phi}\right)\right|=U\left(\frac{W_{1}+W_{2}}{2}\right)=\frac{1}{2}\left(U W_{1}+U W_{2}\right)=\frac{U_{1}+U_{2}}{2}$, where $U_{1}=U W_{1}$ and $U_{2}=U W_{2}$ are the two unitary operators on the Bergman space $A^{2}(\mathbb{D})$. The proof is thus completed.

Corollary 2.2 Let $\|\phi\|_{\infty} \leq 1$, where $\phi \geq 0$ for $\phi \in L^{\infty}(\mathbb{D})$. If $\left\|U_{a}-T_{\phi}\right\|<1, \quad \forall a \in \mathbb{D}$. Then, the Aluthge transform $\Delta\left(T_{\phi \circ \phi_{a}}\right)$ of $T_{\phi \circ \phi_{a}}$ can be written as an average of the two unitaries.

Proof Since $\left\|U_{a}-T_{\phi}\right\|<1$, by ([8], Corollary-1), $T_{\phi}$ is invertible. By ([4], Proposition 3.3), we have $\Delta\left(T_{\phi \circ \phi_{a}}\right)=\Delta\left(U_{a} T_{\phi} U_{a}\right)=U_{a} \Delta\left(T_{\phi}\right) U_{a}$. Hence, the result holds from Theorem 2.1.

Corollary 2.3 Let $\phi$ be an essentially bounded Lebesgue measurable function on $\mathbb{D}$ such that $\phi \geq 0$ and $\|\phi\|_{\infty} \leq 1$. If $\left\|U_{a}-T_{\phi}\right\|<1$, the Aluthge transform $\Delta\left(T_{\phi}\right)$ of $T_{\phi}$ can be represented as $\Delta\left(T_{\phi}\right)=$ $\sum_{j=1}^{4} \frac{(-1)^{j+1}}{4} V_{j}$, where $V_{j}$ is a unitary operator.

Proof Let $\Delta\left(T_{\phi}\right)=U\left|\Delta\left(T_{\phi}\right)\right|$ be the polar decomposition of $\Delta\left(T_{\phi}\right)$. As $\|\phi\|_{\infty} \leq 1$, so $\left\|T_{\phi}\right\| \leq 1$. Given that $\left\|U_{a}-T_{\phi}\right\|<1$, from ([8], Corollary-1), $T_{\phi}$ is invertible. Therefore, $\Delta\left(T_{\phi}\right)$ is also invertible. So $U$ is a unitary operator. It is known that $\left\|\Delta\left(T_{\phi}\right)\right\| \leq\left\|T_{\phi}\right\| \leq 1$. This implies $\left\|\left|\Delta\left(T_{\phi}\right)\right|\right\| \leq 1$. Hence, $I-$ $\left|\Delta\left(T_{\phi}\right)\right|^{2}$ is a positive operator and $\left\|I-\left|\Delta\left(T_{\phi}\right)\right|^{2}\right\| \leq 1$. So we can define the four operators as $W_{1}=$ $\left|\Delta\left(T_{\phi}\right)\right|+i\left(I-\left|\Delta\left(T_{\phi}\right)\right|^{2}\right)^{\frac{1}{2}}, W_{3}=\left|\Delta\left(T_{\phi}\right)\right|-i\left(I-\left|\Delta\left(T_{\phi}\right)\right|^{2}\right)^{\frac{1}{2}}, W_{2}=-\left|\Delta\left(T_{\phi}\right)\right|+i\left(I-\left|\Delta\left(T_{\phi}\right)\right|^{2}\right)^{\frac{1}{2}}$ and $W_{4}=-\left|\Delta\left(T_{\phi}\right)\right|-i\left(I-\left|\Delta\left(T_{\phi}\right)\right|^{2}\right)^{\frac{1}{2}}$. Hence, $W_{1}, W_{2}, W_{3}$ and $W_{4}$ are unitary operators on the Bergman space $A^{2}(\mathbb{D})$. Thus, $\Delta\left(T_{\phi}\right)=U\left|\Delta\left(T_{\phi}\right)\right|=U\left(\frac{W_{1}-W_{2}+W_{3}-W_{4}}{4}\right)=\frac{1}{4}\left(U W_{1}-U W_{2}+U W_{3}-U W_{4}\right)=\frac{V_{1}-V_{2}+V_{3}-V_{4}}{4}$, where $V_{1}=U W_{1}, V_{2}=U W_{2}, V_{3}=U W_{3}$ and $V_{4}=U W_{4}$ are four unitary operators on $A^{2}(\mathbb{D})$.

Corollary 2.4 Let $\phi_{1}, \phi_{2} \in L^{\infty}(\mathbb{D})$ with $\left\|\phi_{1}\right\|_{\infty},\left\|\phi_{2}\right\|_{\infty} \leq 1$ and let $T_{\phi_{1}}=Q_{1}\left|T_{\phi_{1}}\right|$ and $T_{\phi_{2}}=Q_{2}\left|T_{\phi_{2}}\right|$ be the polar decompositions of $T_{\phi_{1}}$ and $T_{\phi_{2}}$, respectively. If $T_{\phi_{1}}$ doubly commutes with $T_{\phi_{2}}$ (that is, $\left[T_{\phi_{1}}, T_{\phi_{2}}\right]=$ 0 and $\left.\left[T_{\phi_{1}}, T_{\phi_{2}}^{*}\right]=0\right)$ and $\operatorname{dim} \operatorname{ker}\left(T_{\phi_{1}} T_{\phi_{2}}\right)=\operatorname{dim} \operatorname{ker}\left(T_{\overline{\phi_{2}}} T_{\overline{\phi_{1}}}\right)$. Then, $\Delta\left(T_{\phi_{1}} T_{\phi_{2}}\right)$ can be represented as $\Delta\left(T_{\phi_{1}} T_{\phi_{2}}\right)=\sum_{j=1}^{4} \frac{(-1)^{j+1}}{4} V_{j}$, where $V_{j}$ is a unitary operator.

Proof Let $T_{\phi_{1}}=Q_{1}\left|T_{\phi_{1}}\right|$ and $T_{\phi_{2}}=Q_{2}\left|T_{\phi_{2}}\right|$ be the polar decompositions of $T_{\phi_{1}}$ and $T_{\phi_{2}}$, respectively, and $T_{\phi_{1}}$ doubly commutes with $T_{\phi_{2}}$ then by [7], $T_{\phi_{1}} T_{\phi_{2}}=Q_{1} Q_{2}\left|T_{\phi_{1}} T_{\phi_{2}}\right|$ is also the polar decomposition of $T_{\phi_{1}} T_{\phi_{2}}$. Further, since $\operatorname{dim} \operatorname{ker}\left(T_{\phi_{1}} T_{\phi_{2}}\right)=\operatorname{dim} \operatorname{ker}\left(T_{\overline{\phi_{2}}} T_{\overline{\phi_{1}}}\right), Q_{1} Q_{2}$ is unitary. Thus, the result is obvious by Corollary 2.3 .

Corollary 2.5 Let $T_{\phi}=U\left|T_{\phi}\right|$ be the polar decomposition of $T_{\phi}$ with the symbol $\phi \in L^{\infty}(\mathbb{D})$ and $\|\phi\|_{\infty} \leq 1$. If $\operatorname{dim} \operatorname{ker}\left(T_{\phi}^{2}\right)=\operatorname{dim} \operatorname{ker}\left(T_{\bar{\phi}}^{2}\right)$, then $\Delta\left(T_{\phi}^{2}\right)$ can be represented as $\frac{1}{4}$ of the alternating finite series of four unitary operators.

Proof Since $T_{\phi}^{2}=U^{2}\left|T_{\phi}^{2}\right|$ is the polar decomposition of $T_{\phi}^{2}$ and $\Delta\left(T_{\phi}^{2}\right)=U^{2}\left|\Delta\left(T_{\phi}^{2}\right)\right|$ is also the polar decomposition of $\Delta\left(T_{\phi}^{2}\right)$, the result follows from Corollary 2.4. 
Corollary 2.6 Let $\|\phi\|_{\infty} \leq 1$ for $\phi \in L^{\infty}(\mathbb{D})$ and $T_{\phi}=U\left|T_{\phi}\right|$ be the polar decomposition of $T_{\phi}$. If $T_{\phi}$, centered with index of $T_{\phi}^{n}$ is equal to zero. Then, $\Delta\left(T_{\phi}^{n}\right)$ can be represented as $\frac{1}{4}$ times the alternating finite series of four unitary operators.

Proof If $T_{\phi}$ is centered then by [10], we obtain $T_{\phi}^{n}=U^{n}\left|T_{\phi}^{n}\right|$ is the polar decomposition for all $n \in \mathbb{N}$. Again $\Delta\left(T_{\phi}^{n}\right)=U^{n}\left|\Delta\left(T_{\phi}^{n}\right)\right|$ is also the polar decomposition of $\Delta\left(T_{\phi}^{n}\right)$. Thus, the proof is evident from Corollary 2.4.

Here, we introduce the tensor product of Toeplitz operators $T_{\phi_{1}} \hat{\otimes} T_{\phi_{2}} \hat{\otimes} \cdots \hat{\otimes} T_{\phi_{n}}$ on tensor product of Bergman spaces $A^{2}(\mathbb{D}) \hat{\otimes} \cdots \hat{\otimes} A^{2}(\mathbb{D})$.

Let $T_{\phi_{j}}=Q_{j}\left|T_{\phi_{j}}\right|$ be the polar decompositions of $T_{\phi_{j}}$ for each $j=1,2, \cdots, n$. Then, $T_{\phi_{1}} \hat{\otimes} T_{\phi_{2}} \hat{\otimes} \cdots \hat{\otimes} T_{\phi_{n}}$ $=\left(Q_{1} \hat{\otimes} Q_{2} \hat{\otimes} \cdots \hat{\otimes} Q_{n}\right)\left|T_{\phi_{1}} \hat{\otimes} T_{\phi_{2}} \hat{\otimes} \cdots \hat{\otimes} T_{\phi_{n}}\right|$ is the polar decomposition of $T_{\phi_{1}} \hat{\otimes} T_{\phi_{2}} \hat{\otimes} \cdots \hat{\otimes} T_{\phi_{n}}$. For more details, see [17].

Corollary 2.7 Let $\phi_{j} \in L^{\infty}(\mathbb{D})$ for $j=1,2, \cdots, n$ such that $\phi_{j} \geq 0$ and $\left\|\phi_{j}\right\|_{\infty} \leq 1$. If $\left\|T_{1+\phi_{j}}\right\|<1$. Then, the Aluthge transform $\Delta\left(T_{\phi_{1}} \hat{\otimes} T_{\phi_{2}} \hat{\otimes} \cdots \hat{\otimes} T_{\phi_{n}}\right)$ of $T_{\phi_{1}} \hat{\otimes} T_{\phi_{2}} \hat{\otimes} \cdots \hat{\otimes} T_{\phi_{n}}$ can be represented as the average of $2^{n}$ unitary operators on $A^{2}(\mathbb{D}) \hat{\otimes} \cdots \hat{\otimes} A^{2}(\mathbb{D})$.

Proof Let there be $\phi_{j} \in L^{\infty}(\mathbb{D}), \phi_{j} \geq 0$ with $\left\|\phi_{j}\right\|_{\infty} \leq 1,\left\|T_{1+\phi_{j}}\right\|<1$ for $j=1,2, \cdots, n$, then by Theorem -2.1 , we find that $\Delta\left(T_{\phi_{j}}\right)$ can be represented as an average of two unitary operators for each $j$. It is known from the properties of Aluthge transform [17] of operators that $\Delta\left(T_{\phi_{1}} \hat{\otimes} T_{\phi_{2}} \hat{\otimes} \cdots \hat{\otimes} T_{\phi_{n}}\right)=$ $\Delta\left(T_{\phi_{1}}\right) \hat{\otimes} \Delta\left(T_{\phi_{2}}\right) \hat{\otimes} \cdots \hat{\otimes} \Delta\left(T_{\phi_{n}}\right)$. Hence, by induction the result is obvious from Theorem -2.1.

For single operator $A \in \mathcal{L}(H)$, we define two elementary operators $L_{A}$ and $R_{A}$ on $\mathcal{L}(H)$ by $L_{A}(X)=A X$ and $R_{A}(X)=X A$ for every $X \in \mathcal{L}(H)$. For more details, see [15]. Here, our immediate corollary is about the Aluthge transform of tensor product of two elementary operators.

Corollary 2.8 Let $T_{\phi_{1}}=Q_{1}\left|T_{\phi_{1}}\right|, T_{\phi_{2}}=Q_{2}\left|T_{\phi_{2}}\right|, \quad T_{\psi_{1}}=R_{1}\left|T_{\psi_{1}}\right|$ and $T_{\psi_{2}}=R_{2}\left|T_{\psi_{2}}\right|$ be the polar decompositions of $T_{\phi_{1}}, T_{\phi_{2}}, T_{\psi_{1}}$ and $T_{\psi_{2}}$, respectively, and let $\left\|\phi_{j}\right\|_{\infty} \leq 1,\left\|\psi_{j}\right\|_{\infty} \leq 1$ for $j=1,2$ and $T_{\phi_{1}}$ doubly commutes with $T_{\phi_{2}}$ and $T_{\psi_{1}}$ doubly commutes with $T_{\psi_{2}}$. If $\operatorname{dim} \operatorname{ker}\left(T_{\phi_{1}} T_{\phi_{2}}\right)=\operatorname{dim} \operatorname{ker}\left(T_{\overline{\phi_{2}}} T_{\overline{\phi_{1}}}\right)$ and $\operatorname{dim} \operatorname{ker}\left(T_{\psi_{1}} T_{\psi_{2}}\right)=\operatorname{dim} \operatorname{ker}\left(T_{\overline{\psi_{2}}} T_{\overline{\psi_{1}}}\right)$. Then, the Aluthge transform

$$
\Delta\left(L_{T_{\phi_{1}}}\left(T_{\phi_{2}}\right) \hat{\otimes} R_{T_{\psi_{2}}}\left(T_{\psi_{1}}\right)\right) \text { of } L_{T_{\phi_{1}}}\left(T_{\phi_{2}}\right) \hat{\otimes} R_{T_{\psi_{2}}}\left(T_{\psi_{1}}\right)
$$

is the average of four unitary operators.

Proof Since $T_{\phi_{1}}$ doubly commutes with $T_{\phi_{2}}$ and $T_{\psi_{1}}$ doubly commutes with $T_{\psi_{2}}$ then by [7], $T_{\phi_{1}} T_{\phi_{2}}=$ $Q_{1} Q_{2}\left|T_{\phi_{1}} T_{\phi_{2}}\right|$ and $T_{\psi_{1}} T_{\psi_{2}}=R_{1} R_{2}\left|T_{\psi_{1}} T_{\psi_{2}}\right|$ are the polar decompositions of $T_{\phi_{1}} T_{\phi_{2}}$ and $T_{\psi_{1}} T_{\psi_{2}}$, respectively. Further, as $\Delta\left(T_{\phi_{1}} T_{\phi_{2}}\right)=Q_{1} Q_{2}\left|\Delta\left(T_{\phi_{1}} T_{\phi_{2}}\right)\right|$ and $\Delta\left(T_{\psi_{1}} T_{\psi_{2}}\right)=R_{1} R_{2}\left|\Delta\left(T_{\psi_{1}} T_{\psi_{2}}\right)\right|$ are the polar decompositions of $T_{\phi_{1}} T_{\phi_{2}}$ and $T_{\psi_{1}} T_{\psi_{2}}$, respectively. Thus, $T_{\phi_{1}} T_{\phi_{2}} \hat{\otimes} T_{\psi_{1}} T_{\psi_{2}}=\left(Q_{1} Q_{2} \hat{\otimes} R_{1} R_{2}\right)\left|T_{\phi_{1}} T_{\phi_{2}} \hat{\otimes} T_{\psi_{1}} T_{\psi_{2}}\right|$ is also the polar decomposition of $T_{\phi_{1}} T_{\phi_{2}} \hat{\otimes} T_{\psi_{1}} T_{\psi_{2}}$. It is clear from the properties of Aluthge transform of an operator that $\Delta\left(T_{\phi_{1}} T_{\phi_{2}} \hat{\otimes} T_{\psi_{1}} T_{\psi_{2}}\right)=\Delta\left(T_{\phi_{1}} T_{\phi_{2}}\right) \hat{\otimes} \Delta\left(T_{\psi_{1}} T_{\psi_{2}}\right)$. Given that $\operatorname{dim} \operatorname{ker}\left(T_{\phi_{1}} T_{\phi_{2}}\right)=\operatorname{dim} \operatorname{ker}\left(T_{\overline{\phi_{2}}} T_{\overline{\phi_{1}}}\right)$ and $\operatorname{dim} \operatorname{ker}\left(T_{\psi_{1}} T_{\psi_{2}}\right)=\operatorname{dim} \operatorname{ker}\left(T_{\overline{\psi_{2}}} T_{\overline{\psi_{1}}}\right)$, this implies $Q_{1} Q_{2}$ and $R_{1} R_{2}$ are unitary operators. Now,

$$
\begin{aligned}
\left\|\Delta\left(T_{\phi_{1}} T_{\phi_{2}} \hat{\otimes} T_{\psi_{1}} T_{\psi_{2}}\right)\right\| & =\left\|\Delta\left(T_{\phi_{1}} T_{\phi_{2}}\right) \hat{\otimes} \Delta\left(T_{\psi_{1}} T_{\psi_{2}}\right)\right\| \\
& \leq\left\|T_{\phi_{1}} T_{\phi_{2}}\right\|\left\|T_{\psi_{1}} T_{\psi_{2}}\right\| \\
& \leq 1 .
\end{aligned}
$$

Hence, the result is evident from Theorem 2.1.

\section{Normal operator}

In this section, we state some sufficient conditions for Aluthge transform of tensor product of Toeplitz and little Hankel operators to be normal.

Theorem 3.1 Let $\phi \geq 0, \psi \geq 0 \in L^{\infty}(\mathbb{D})$ be such that $\|1+\phi\|_{\infty}<1$ and $\|1+\psi\|_{\infty}<1$. Suppose that $T_{\phi}=U\left|T_{\phi}\right|$ and $S_{\psi}=V\left|S_{\psi}\right|$ be the polar decompositions of $T_{\phi}$ and $S_{\psi}$ on $A^{2}(\mathbb{D})$, respectively, with $s p(U)$ and $\operatorname{sp}(V)$ are contained in some open semi-circles. Then, $\Delta\left(T_{\phi} \hat{\otimes} S_{\psi}\right)$ is normal iff $T_{\phi}, S_{\psi}$ are normal. 
Proof Since $\phi, \psi \geq 0$ then $T_{\phi}$ and $S_{\psi}$ are positive on $A^{2}(\mathbb{D})$. Thus by ([1], theorem-3.1), for every unitary operators $W_{1}$ and $W_{2}$ on $A^{2}(\mathbb{D})$ we have $\left\|W_{1}-T_{\phi}\right\| \leq\left\|I+T_{\phi}\right\|=\left\|T_{1+\phi}\right\| \leq\|1+\phi\|_{\infty}<1$ and $\left\|W_{2}-S_{\psi}\right\| \leq\left\|I+S_{\psi}\right\|=\left\|S_{1+\psi}\right\| \leq\|1+\psi\|_{\infty}<1$. Hence, $T_{\phi}$ and $S_{\psi}$ are invertible. Since $s p(U)$ contained in some open semi-circle then by [11], $\Delta\left(T_{\phi}\right)$ is normal iff $T_{\phi}$ is normal. Similarly using the same argument for $S_{\psi}$, We get $\Delta\left(S_{\psi}\right)$ is normal iff $S_{\psi}$ is normal. As $\Delta\left(T_{\phi} \hat{\otimes} S_{\psi}\right)=\Delta\left(T_{\phi}\right) \hat{\otimes} \Delta\left(S_{\psi}\right)$ for $T_{\phi}, S_{\psi}$ $\in \mathcal{L}^{\infty}(\mathbb{D})$. Therefore, by ([12], Proposition-5), $\Delta\left(T_{\phi} \hat{\otimes} S_{\psi}\right)$ is normal iff $T_{\phi}$ and $S_{\psi}$ are normal.

Corollary 3.2 Let $\phi, \psi \in L^{\infty}(\mathbb{D})$ be such that $\phi, \psi \geq 0$ and $\|1+\phi\|_{\infty}<1$ and $\|1+\psi\|_{\infty}<1$. If $T_{\phi}$ and $S_{\psi}$ are hyponormal operators with spectrum of $T_{\phi}$ and $S_{\psi}$ are arcs, respectively, then $\Delta\left(T_{\phi} \hat{\otimes} S_{\psi}\right)$ is normal.

Proof As $T_{\phi}$ and $S_{\psi}$ are hyponormal with their spectrums as arcs, $T_{\phi}$ and $S_{\psi}$ are normal. Since $T_{\phi}$ is invertible, so by [3], $T_{\phi}$ and $\Delta\left(T_{\phi}\right)$ are similar operators. Similarly, $S_{\psi}$ and $\Delta\left(S_{\psi}\right)$ are similar operators. Again by [3], $\left|T_{\phi}\right|$ and $\left|S_{\psi}\right|$ are invertible as $T_{\phi}$ and $S_{\psi}$ are invertible. Therefore, $T_{\phi}$ and $S_{\psi}$ can be expressed as $\left|T_{\phi}\right|^{-\frac{1}{2}} \Delta\left(T_{\phi}\right)\left|T_{\phi}\right|^{\frac{1}{2}}$ and $\left|S_{\psi}\right|^{-\frac{1}{2}} \Delta\left(S_{\psi}\right)\left|S_{\psi}\right|^{\frac{1}{2}}$, respectively. Hence

$$
T_{\phi}^{*} T_{\phi}=\left|T_{\phi}\right|^{-\frac{1}{2}} \Delta^{*}\left(T_{\phi}\right) \Delta\left(T_{\phi}\right)\left|T_{\phi}\right|^{\frac{1}{2}}
$$

Similarly

$$
T_{\phi} T_{\phi}^{*}=\left|T_{\phi}\right|^{-\frac{1}{2}} \Delta\left(T_{\phi}\right) \Delta^{*}\left(T_{\phi}\right)\left|T_{\phi}\right|^{\frac{1}{2}}
$$

As $T_{\phi}$ is normal, it is clear from equations (3.1) and (3.2) that $\Delta\left(T_{\phi}\right)$ is normal. Similarly, $\Delta\left(S_{\psi}\right)$ is normal. Thus, by Theorem 3.1, $\Delta\left(T_{\phi} \hat{\otimes} S_{\psi}\right)$ is normal.

Corollary 3.3 Let $T_{\phi}=U\left|T_{\phi}\right|$ and $S_{\psi}=V\left|S_{\psi}\right|$ be the polar decompositions of $T_{\phi}$ and $S_{\psi}$, respectively. If $U$ and $V$ are hyponormal with $s p(U)$ and $s p(V)$ as the set of real numbers, then $\Delta\left(T_{\phi} \hat{\otimes} S_{\psi}\right)$ is normal.

Proof Let $T_{\phi}=U\left|T_{\phi}\right|$ and $S_{\psi}=V\left|S_{\psi}\right|$ be the polar decomposition of $T_{\phi}$ and $S_{\psi}$, respectively. Now

$$
\begin{aligned}
\Delta^{*}\left(T_{\phi} \hat{\otimes} S_{\psi}\right) & =\left|T_{\phi} \hat{\otimes} S_{\psi}\right|^{\frac{1}{2}}\left(U^{*} \hat{\otimes} V^{*}\right)\left|T_{\phi} \hat{\otimes} S_{\psi}\right|^{\frac{1}{2}} \\
& =\left(\left|T_{\phi}\right|^{\frac{1}{2}} U^{*}\left|T_{\phi}\right|^{\frac{1}{2}}\right) \hat{\otimes}\left(\left|S_{\psi}\right|^{\frac{1}{2}} V^{*}\left|S_{\psi}\right|^{\frac{1}{2}}\right)
\end{aligned}
$$

As $U$ and $V$ are hyponormal and $s p(U), s p(V)$ are the set of real numbers. So by [16], $U$ and $V$ are self-adjoint. Therefore

$$
\begin{aligned}
\Delta^{*}\left(T_{\phi} \hat{\otimes} S_{\psi}\right) & =\left(\left|T_{\phi}\right|^{\frac{1}{2}} U\left|T_{\phi}\right|^{\frac{1}{2}}\right) \hat{\otimes}\left(\left|S_{\psi}\right|^{\frac{1}{2}} V\left|H_{\psi}\right|^{\frac{1}{2}}\right) \\
& =\Delta\left(T_{\phi} \hat{\otimes} S_{\psi}\right)
\end{aligned}
$$

So $\Delta\left(T_{\phi} \hat{\otimes} S_{\psi}\right)$ is self-adjoint. Since self-adjoint operators are normal, so $\Delta\left(T_{\phi} \hat{\otimes} S_{\psi}\right)$ is normal.

Theorem 3.4 Let $\phi, \psi \in L^{\infty}(\mathbb{D})$ and $X \in \mathcal{L}\left(A^{2}(\mathbb{D})\right)$. If $\phi$ and $\psi \geq 0$ with $T_{\phi} X=I$ and $S_{\psi} X=I$ then $\Delta\left(T_{\phi} \hat{\otimes} S_{\psi}\right)$ is normal.

Proof As $\phi$ and $\psi$ are positive, $T_{\phi}$ and $S_{\psi}$ are positive on $A^{2}(\mathbb{D})$. Since $T_{\phi} X=I, T_{\phi}$ is right invertible. Now $X^{*} T_{\phi}=\left(T_{\phi} X\right)^{*}=I^{*}=I$. This implies that $T_{\phi}$ is left invertible. Hence, $T_{\phi}$ is invertible. Similarly, $S_{\psi}$ is invertible. Then by [5], $\Delta\left(T_{\phi}\right), \Delta\left(S_{\psi}\right)$ are positive. Since positive operators are normal, then $\Delta\left(T_{\phi}\right), \Delta\left(S_{\psi}\right)$ are normal. Then by [12], $\Delta\left(T_{\phi} \hat{\otimes} S_{\psi}\right)$ is normal.

Theorem 3.5 Let $T_{\phi}, S_{\psi} \in \mathcal{L}\left(A^{2}(\mathbb{D})\right)$ with $m\left(T_{\phi}\right), m\left(T_{\phi}^{*}\right)>0$ and $m\left(S_{\psi}\right), m\left(S_{\psi}^{*}\right)>0$. If $T_{\phi}$ and $S_{\psi}$ are partial isometry satisfying $T_{\phi}^{* 2} T_{\phi}^{2}+2 k T_{\phi}^{*} T_{\phi}+k^{2} \geq 0$ and $S_{\psi}^{* 2} S_{\psi}^{2}+2 k S_{\psi}^{*} S_{\psi}+k^{2} \geq 0$ for all real $k$ then $\Delta\left(T_{\phi} \hat{\otimes} S_{\psi}\right)$ is normal.

Proof Let $m\left(T_{\phi}\right)=\inf \left\{\left\|T_{\phi} x\right\|:\|x\|=1\right\}$ and $m\left(T_{\phi}^{*}\right)=\inf \left\{\left\|T_{\phi}^{*} x\right\|:\|x\|=1\right\}$. Since $m\left(T_{\phi}\right), m\left(T_{\phi}^{*}\right)>0$ then by [18], $T_{\phi}$ is invertible. Similarly, $S_{\psi}$ is invertible. Again since $T_{\phi}^{* 2} T_{\phi}^{2}+2 k T_{\phi}^{*} T_{\phi}+k^{2} \geq 0$ and $S_{\psi}^{* 2} S_{\psi}^{2}+2 k S_{\psi}^{*} S_{\psi}+k^{2} \geq 0$ for any real number k, so $T_{\phi}$ and $S_{\psi}$ are paranormal. As partial isometry and 
paranormal operators are quasinormal. So $T_{\phi}$ and $S_{\psi}$ are quasinormal. Therefore by [13], $\Delta\left(T_{\phi}\right)=T_{\phi}$ and $\Delta\left(S_{\psi}\right)=S_{\psi}$. Thus,

$$
\begin{aligned}
\Delta\left(T_{\phi} \hat{\otimes} S_{\psi}\right) & =\Delta\left(T_{\phi}\right) \hat{\otimes} \Delta\left(S_{\psi}\right) \\
& =T_{\phi} \hat{\otimes} S_{\psi} .
\end{aligned}
$$

Since $T_{\phi}$ and $S_{\psi}$ are quasinormal and invertible operators, this implies that $T_{\phi}$ and $S_{\psi}$ are normal. Hence by [12], $\Delta\left(T_{\phi} \hat{\otimes} S_{\psi}\right)$ is normal.

Corollary 3.6 Let $T_{\phi}=T_{\phi_{1}} T_{\phi_{2}}, S_{\psi}=S_{\psi_{1}} S_{\psi_{2}}$. If $T_{\phi_{1}}, T_{\phi_{2}}, S_{\psi_{1}}$ and $S_{\psi_{2}}$ are invertible quasinormal operators with $\left[T_{\phi_{1}}, T_{\phi_{2}}\right]=\left[T_{\phi_{1}}, T_{\phi_{2}}^{*}\right]=0$ and $\left[S_{\psi_{1}}, S_{\psi_{2}}\right]=\left[S_{\psi_{1}}, S_{\psi_{2}}^{*}\right]=0$ then $\Delta\left(T_{\phi} \hat{\otimes} S_{\psi}\right)$ is normal.

Proof Since $T_{\phi_{1}}$ and $T_{\phi_{2}}$ are invertible quasinormal operators and also $\left[T_{\phi_{1}}, T_{\phi_{2}}\right]=\left[T_{\phi_{1}}, T_{\phi_{2}}^{*}\right]=0$ then by [9], $T_{\phi_{1}} T_{\phi_{2}}$ is normal. Similarly, $S_{\psi_{1}} S_{\psi_{2}}$ is normal. Now

$$
\Delta\left(\left(T_{\phi_{1}} T_{\phi_{2}}\right) \hat{\otimes}\left(S_{\psi_{1}} S_{\psi_{2}}\right)\right)=\Delta\left(T_{\phi_{1}} T_{\phi_{2}}\right) \hat{\otimes} \Delta\left(S_{\psi_{1}} S_{\psi_{2}}\right) .
$$

As $T_{\phi_{1}}$ and $T_{\phi_{2}}$ are quasinormal with $T_{\phi_{1}} T_{\phi_{2}}=T_{\phi_{2}} T_{\phi_{1}}$ and $T_{\phi_{1}} T_{\phi_{2}}^{*}=T_{\phi_{2}}^{*} T_{\phi_{1}}$ then $T_{\phi_{1}} T_{\phi_{2}}$ is quasinormal. Similarly, $S_{\psi_{1}} S_{\psi_{2}}$ is quasinormal. Hence by [13], $\Delta\left(T_{\phi_{1}} T_{\phi_{2}}\right)=T_{\phi_{1}} T_{\phi_{2}}$ and $\Delta\left(S_{\psi_{1}} S_{\psi_{2}}\right)=S_{\psi_{1}} S_{\psi_{2}}$. Therefore, from Eq. (3.3), we obtained

$$
\begin{aligned}
\Delta\left(\left(T_{\phi_{1}} T_{\phi_{2}}\right) \hat{\otimes}\left(S_{\psi_{1}} S_{\psi_{2}}\right)\right) & =\Delta\left(T_{\phi_{1}} T_{\phi_{2}}\right) \hat{\otimes} \Delta\left(S_{\psi_{1}} S_{\psi_{2}}\right) \\
& =T_{\phi_{1}} T_{\phi_{2}} \hat{\otimes} S_{\psi_{1}} S_{\psi_{2}} .
\end{aligned}
$$

Thus, the proof is evident from [12].

Theorem 3.7 Let $\phi=\phi_{1}+\phi_{2} \in L^{\infty}(\mathbb{D})$ and $\psi=\psi_{1}+\psi_{2} \in L^{\infty}(\mathbb{D})$ are positive measurable functions. Suppose $T_{\phi}, S_{\psi}$ are invertible operators with $\left[\Delta\left(T_{\phi_{1}}\right), \Delta\left(T_{\phi_{2}}^{*}\right)\right]=0$ and $\left[\Delta\left(S_{\psi_{1}}\right), \Delta\left(S_{\psi_{2}}^{*}\right)\right]=0$ then $\Delta\left(T_{\phi} \hat{\otimes} S_{\psi}\right)$ is normal.

Proof Since $\phi$ and $\psi \geq 0$ then $T_{\phi}, S_{\psi}$ are positive operators. Therefore

$$
T_{\phi}=T_{\phi_{1}}+T_{\phi_{2}} \quad \text { and } \quad S_{\psi}=S_{\psi_{1}}+S_{\psi_{2}}
$$

are positive. So $T_{\phi_{1}}, T_{\phi_{2}}$ are positive and as $T_{\phi_{1}}, T_{\phi_{2}}$ are invertible operators, By [5], $\Delta\left(T_{\phi_{1}}\right)$ and $\Delta\left(T_{\phi_{2}}\right)$ are positive. Since positive operators are normal. So $\Delta\left(T_{\phi_{1}}\right)$ and $\Delta\left(T_{\phi_{2}}\right)$ are normal. Similarly, we can easily seen that $\Delta\left(S_{\psi_{1}}\right)$ and $\Delta\left(S_{\psi_{2}}\right)$ are normal. Therefore

$$
\begin{aligned}
\Delta\left(T_{\phi} \hat{\otimes} S_{\psi}\right) & =\Delta\left(T_{\phi}\right) \hat{\otimes} \Delta\left(S_{\psi}\right) \\
& =\Delta\left(T_{\phi_{1}}+T_{\phi_{2}}\right) \hat{\otimes} \Delta\left(S_{\psi_{1}}+S_{\psi_{2}}\right) \\
& =\left(\Delta\left(T_{\phi_{1}}\right)+\Delta\left(T_{\phi_{2}}\right)\right) \hat{\otimes}\left(\Delta\left(S_{\psi_{1}}\right)+\Delta\left(S_{\psi_{2}}\right)\right) .
\end{aligned}
$$

Further, since $\left[\Delta\left(T_{\phi_{1}}\right), \Delta\left(T_{\phi_{2}}^{*}\right)\right]=0$ and $\left[\Delta\left(S_{\psi_{1}}\right), \Delta\left(S_{\psi_{2}}^{*}\right)\right]=0, \Delta\left(T_{\phi_{1}}\right)+\Delta\left(T_{\phi_{2}}\right)$ and $\Delta\left(S_{\psi_{1}}\right)+\Delta\left(S_{\psi_{2}}\right)$ are normal. Hence, $\Delta\left(T_{\phi} \hat{\otimes} S_{\psi}\right)$ is normal.

Theorem 3.8 Let $T_{\phi}=T_{\phi_{1}}+i T_{\phi_{2}}$ and $S_{\psi}=S_{\psi_{1}}+i S_{\psi_{2}}$ be the cartesian decompositions of $T_{\phi}$ and $S_{\psi}$, respectively. Suppose $T_{\phi}$ and $S_{\psi}$ are invertible operators. If $T_{\phi}, S_{\psi}$ are hyponormal operators and $T_{\phi_{2}}, S_{\psi_{2}}$ are compact, then $\Delta\left(T_{\phi} \hat{\otimes} S_{\psi}\right)$ is normal.

Proof Let $T_{\phi}=T_{\phi_{1}}+i T_{\phi_{2}}$ and $S_{\psi}=S_{\psi_{1}}+i S_{\psi_{2}}$ be the cartesian decompositions of $T_{\phi}$ and $S_{\psi}$, respectively. Since $T_{\phi}, S_{\psi}$ are hyponormal operators whose imaginary parts are compact, so by [19], $T_{\phi}, S_{\psi}$ are normal. Again, since $T_{\phi}$ and $S_{\psi}$ are invertible, therefore by [3], $T_{\phi}$ and $\Delta\left(T_{\phi}\right)$ are similar operators. Similarly, $S_{\psi}$ and $\Delta\left(S_{\psi}\right)$ are similar operators and also $\left|T_{\phi}\right|$ and $\left|S_{\psi}\right|$ are invertible as $T_{\phi}$ and $S_{\psi}$ are invertible. Therefore, we can express $T_{\phi}$ and $S_{\psi}$ as $T_{\phi}=\left|T_{\phi}\right|^{\frac{-1}{2}} \Delta\left(T_{\phi}\right)\left|T_{\phi}\right|^{\frac{1}{2}}$ and $S_{\psi}=\left|S_{\psi}\right|^{\frac{-1}{2}} \Delta\left(S_{\psi}\right)\left|S_{\psi}\right|^{\frac{1}{2}}$, respectively. So, as $T_{\phi}$ and $S_{\psi}$ are normal it can be easily shown that $\Delta\left(T_{\phi}\right)$ and $\Delta\left(S_{\psi}\right)$ are normal. Hence, $\Delta\left(T_{\phi} \hat{\otimes} S_{\psi}\right)$ is normal.

Open Access This article is distributed under the terms of the Creative Commons Attribution 4.0 International License (http:// creativecommons.org/licenses/by/4.0/), which permits unrestricted use, distribution, and reproduction in any medium, provided you give appropriate credit to the original author(s) and the source, provide a link to the Creative Commons license, and indicate if changes were made. 


\section{References}

1. Aiken, J.G.; Erdos, J.A.; Goldstein, J.A.: Unitary approximation of positive operators. Illinois J. Math. 24, 61-72 (1980)

2. Aluthge, A.: On p-hyponormal operators for $0<p<1$. Integr. Equ. Oper. Theory 13, 307-315 (1990)

3. Aluthge, A.; Wang, D.: W-hyponormal operators. Integr. Equ. Oper. Theory 36, 1-10 (2000)

4. Antezana, J.; Massey, P.; Stojanoff, D.: $\lambda$-Aluthge transforms and Schatten ideals. Linear Algebra Appl. 405, 177-199 (2005)

5. Chabbabi, F.: product commuting maps with the $\lambda$ aluthge transform (2016). arXiv:1606.06165v2 [math.FA]

6. Das, N.; Jena, P.K.: On the range and kernel Of Toeplitz and little Hankel operators. Methods Funct. Anal. Topol. 19, 55-67 (2013)

7. Furuta, T.: On the polar decomposition of an operator. Acta Sci. Math. (Szeged) 46, 261-268 (1983)

8. Lin, C.S.: The unilateral shift and a norm equality for bounded linear operators. Proc. Am. Math. Soc. 127, 1693-1696 (1999)

9. Imagiri, S.K.; Khalagai, J.M.; Pokariyal, G.P.: On the normality of the product of n-power quasinormal operators. Far East J. Appl. Math. 82(1), 41-53 (2013)

10. Morrel, B.B.; Muhly, P.S.: Centered operators. Studia Math. 51, 251-263 (1974)

11. Moslehian, M.S., Nabavi Sales, S.M.S.: Some conditions implying normality of operators, C. R. Math. 349(5-6), 251-254 (2011)

12. Kubrusly, C.S.: Concise introduction to tensor product. Far East J. Math. Sci. 22, 137-171 (2006)

13. Jung, I.B.; Ko, E.; Pearcy, C.: Aluthge transforms of operators. Integr. Equ. Oper. Theory 37, 437-448 (2000)

14. Olsen, C.L.: Unitary approximation. J. Funct. Anal. 85, 392-419 (1989)

15. Seddik, A.: Rank one operators and norm of elementary operators. Linear Algebra Appl. 424, 177-183 (2007)

16. Stampfli, J.G.: Hyponormal operators and Spectral density. Trans. Am. Math. Soc. 117, 496-476 (1965)

17. Tanahashi, K.; Cho, M.: Tensor products of log-hyponormal and of class $A(s, t)$ operators. Glasg. Math. J. 46, 91-95 (2004)

18. Wu, P.Y.: Approximation by invertible and noninvertible operators. J. Approx. Theory 56, 267-276 (1989)

19. Whitley, R.: A note on hyponormal operators. Proc. Am. Math. Soc. 49(2), 399-400 (1975)

20. Zhu, K.: Operator Theory in Function Spaces. Marcel Dekker, New York (1990)

Publisher's Note Springer Nature remains neutral with regard to jurisdictional claims in published maps and institutional affiliations. 